\title{
Status of Forward Physics Projects at CMS
}

\author{
Kerstin Borras \\ on behalf of the CMS Collaboration \\ Deutsches Elektronen-Synchrotron (DESY) \\ Notkestrasse 85, 22603 Hamburg - Germany
}

\begin{abstract}
New experiments with detectors in the forward region with an unprecedented coverage of phase space are on the verge of opening a new eara for forward physics at the high energy frontier. The detector components of the CMS experiment are described as well as briefly the components of the TOTEM experiment. The physics topics achievable with these special detectors are outlined and in few cases discussed in more detail.
\end{abstract}

\section{Introduction}

Forward physics is one of the rare high energy physics topics with a very long tradition dating back already to the beginnings of hadron hadron scattering. The reason can be found in the large cross section for soft hadronic interactions, which leads to plenty of data. That forward physics is still a topic for intensive experimental and theoretical studies lies in its predominantly soft structure, which cannot yet be described with the methods of QCD needing a hard scale for perturbative calculations. A lot of progress in the understanding has been achieved by the analysis of semi-hard hadronic interactions, in which the processes still show the characteristic signatures of soft hadronic interactions, like diffraction, but in addition a hard scale is provided by the production of high $p_{T}$ jets or $D^{*}$, being looked at with the data of the running accelerators: $e p$ at HERA, $p p$ at TEVATRON or $p p$ at RHIC. In order to understand the questions opened by these data (and even older data) and to pursue the physics understanding into the new kinematic regime of the LHC, several detectors are planned to complement the coverage of the ATLAS and the CMS experiment in the forward region.

\section{Detectors in the Forward Region of the CMS Interaction Point}

The Interaction Point 5 is the host of the experimental setup for two collaborations as displayed in Figure 1. The CMS detector [2] covers the interaction point with tracking devices up to roughly $|\eta|<2.5$. This is complemented by calorimetry up to $|\eta|<3$ and is further extended by the Hadronic Forward calorimeter (HF) in the region of $3<|\eta|<5$, by the CASTOR calorimeter in $(5.3<|\eta|<6.6)$ and for neutral particles by the Zero Degree Calorimeter (ZDC) starting at about $|\eta|>8$.

The detectors of the TOTEM experiment [3] complement this calorimetric coverage with tracking stations (T1 and T2) and with the installation of Roman Pot stations at $147 \mathrm{~m}$ and $220 \mathrm{~m}$ away from the interaction point.

In addition within the CMS collaboration discussions for the installation of forward proton tagging devices at $420 \mathrm{~m}$ as proposed and studied by the FP420 Collaboration [4], a joint effort with members from CMS, ATLAS and independent physicists, are underway.

In total this results in an unprecedented coverage of the forward region at hadron colliders and a large variety of phyiscs topics which can be studied with these devices. 

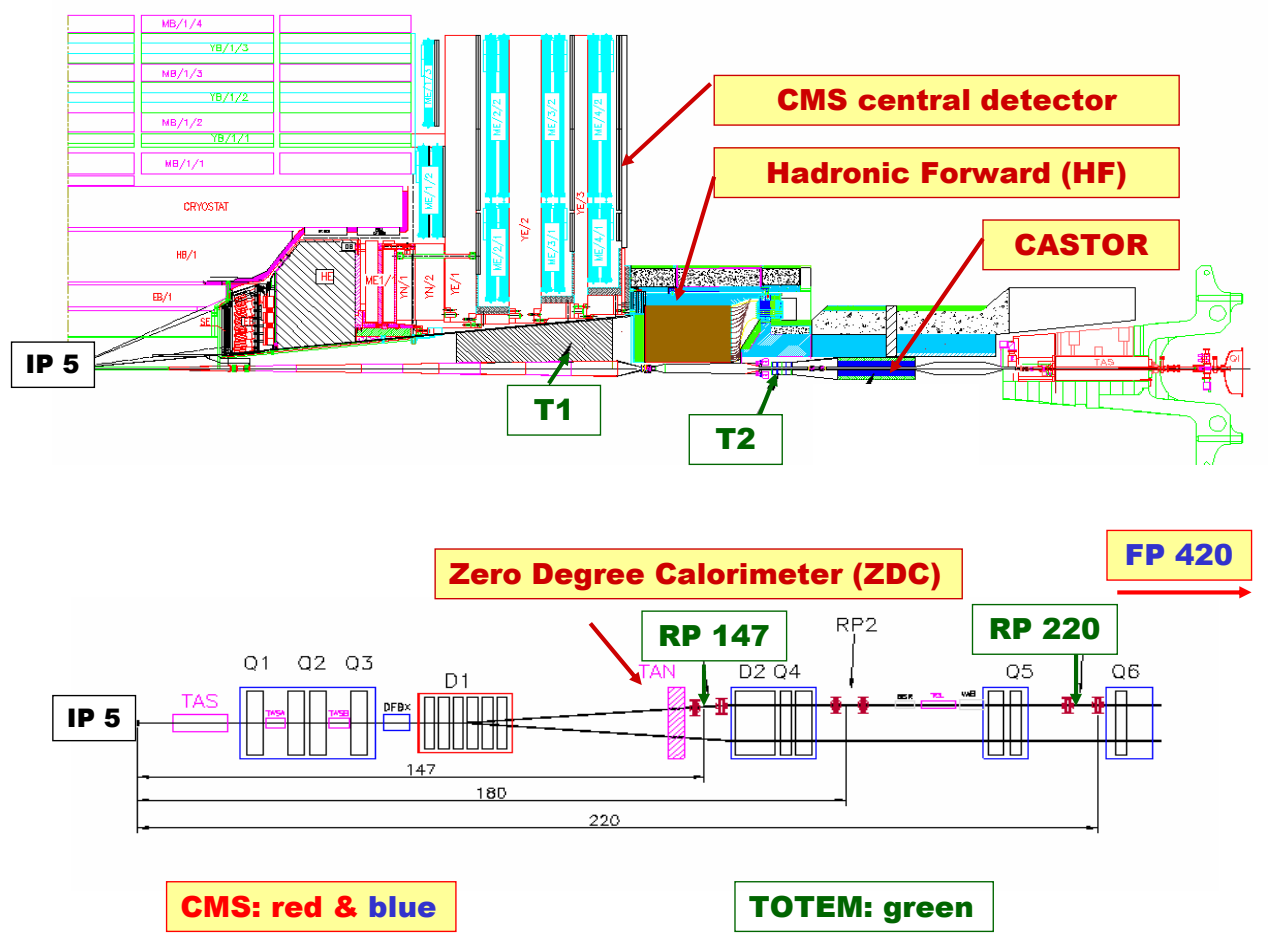

Figure 1: Region around the Interaction Point 5

\section{Physics Program}

The forward physics program spans a broad range of high energy physics topics from fundamental properties of QCD to new physics phenomena and the precise determination of luminosity. These aspects constitute important ingredients for the discovery of new phenomena and their interpretation, like the crucial understanding of the underlying event structure and multi-parton interactions.

Through the AGK cutting rules [5] three main areas for forward physics are closely related: diffraction, saturation at small $\mathrm{x}$ and multi-parton interactions. The AGK cutting rules, steming from early soft hadronic interaction analyses are now under study towards their formulation within the framework of QCD [6].

The prospects for diffractive and forward physics using the devices from the CMS and the TOTEM experiments are summarized in a common CMS and TOTEM note [8]. The experimental issues, the possible measurements and their achievable accuracy for several phyiscs analyses have been studied in more detail in this note. 
The following list gives an overview on most of the possible topics of forward physics [7]:

- Diffraction:

- soft diffraction: rapidity gap survival dynamics, multi-gap events

- hard diffraction: prodcution of jets, $W, J / \Psi, b, t$, hard photons, study of the partonic structure of the diffractive exchange

- double pomeron exchange events (gluon factory)

- diffractive Higgs production

- SUSY and other (low mass) exotics and exclusive processes.

The different experimental observables for diffraction are the tagging of forward scattered protons and the detection of so-called rapidity gaps, areas in $\eta$ devoid of particle production.

Experimental challenges are given by the trigger thresholds and at higher luminosities by pile-up of events. Diffractive events with a hard scale constitute only a very small fraction of the total high $p_{T}$ events. Therefore low trigger thresholds are desirable. For the CMS jet trigger, the typical dijet threshold is about $100 \mathrm{GeV}$ per jet at luminosities around $10^{33} \mathrm{~cm}^{-2} \mathrm{~s}^{-1}$. These can be lowered substantially by adding a request for a track in a Roman Pot of TOTEM at 220m or in FP420. Assuming only about $1 \%$ of the total trigger bandwidth for such a dedicated forward detector stream, requiring dijets in combination with a track in the Roman Pots at $220 \mathrm{~m}$ on one side of the interaction point would allow to lower the dijet threshold to about $40 \mathrm{GeV}$.

For central exclusive production of a light Higgs with $120 \mathrm{GeV}$ mass, this would results in a signal efficiency of approximately $10 \%$ from the jet trigger, to which another $10 \%$ efficiency can be added by triggering on one jet and one muon from the decay of one of the two $\mathrm{b}$ quarks. On the Higher Level Trigger, the correlation between the proton momentum loss $\xi$ as measured by the near beam detectors at $220 \mathrm{~m}$ or $420 \mathrm{~m}$, is a powerful tool to further reduce background from non-diffractive QCD dijet production.

- Low-x dynamics:

- proton structure function, DGLAP/BFKL/CCFM evolution, parton saturation Special studies employ jets or Drell-Yan lepton pairs in the forward detectors.

- Multi-parton interactions and underlying event structure:

In hadron-hadron collisions not only one hard parton-parton interaction can occur, but also additional soft interactions between the partons of the remnants of the beam particles and more hard interactions between the remaining partons. The soft interactions produce a higher energy level in the underlying event, which has to be subtracted when going back from the detector and hadron level to the parton level. The additional hard interactions can lead with standard processes to the same final state particles as looked for in new physics signatures. The final state in $p p \rightarrow W+H+X \rightarrow l+\nu+b \bar{b}+X$ can be produced with double parton interactions in one proton-proton collision with $p p \rightarrow W+X_{W}+b \bar{b}+X_{b}$ without any Higgs production. The contribution from multiple-parton interactions have to be understood before the new physics signal can be unfolded from the measurements.

Predictions for the underlying event and multiple interactions vary quite a lot. In preliminary generator level studies it has been seen, that the forward region is especially sensitive in energy and mean charged particle flow to the different models for underlying event structure and multi-parton interactions [9]. 
- Measurements for Cosmic Ray Physics Model validation: At the LHC, energies corresponding to the order of $100 \mathrm{PeV}$ in the fixed target frame are available and the analysis of energy and particle flow in the forward detectors will provide distinctive information for the validation of hadronic shower modeling in cosmic ray data.

- Photon-proton and photon-photon physics

- Forward physics in heavy ion (pA and AA) collisions

- parton saturation, new phenomena (Centauro's, Strangelets...)

- Luminosity determination with QED processes $(p p \rightarrow p p e e, p p \rightarrow p p \mu \mu)$

The study of elastic scattering and a precise measurement of the total cross section will be performed by the TOTEM collaboration.

In summary the CMS forward detectors provide the possibility for a rich program of forward physics. In conjunction with the tracking devices of TOTEM and FP420 the program comprises a large variety of physics topics for low luminosities, for example with rapidity gap selection for diffraction and underlying event structure/multi-parton interaction studies without pile-up, up to highest luminosities with discovery potential through central exclusive production of light Higgs or SUSY particles.

\section{Acknowledgments}

It is a pleasure to thank the organizers for the enjoyable conference. Special thanks are going to Monika Grothe and Hannes Jung for detailed discussions.

\section{References}

[1] Slides: http://indico.cern.ch/contributionDisplay $\cdot$ py?contribId=98\&sessionId=7\&conf Id=9499

[2] CMS Collaboration, The CMS Physics Technical Design Report, Volume 1, CERN-LHCC-2006-001 (2006).

[3] TOTEM Collaboration, Technical Design Report, CERN-LHCC-2004-002 (2004), and references therein.

[4] FP420 Collaboration, FP420: A proposal to investigate the feasibility of installing proton tagging detectors in the 420m region of the LHC, CERN-LHCC-2005-025 (2005). and A. Pilkington in this conference: http: //indico. cern. ch/contributionDisplay.py?contribId=99\&sessionId=7\&conf Id=9499

[5] V.A. Abramowsky, V.N. Gribov, O.V. Kancheli, Sov. J. Nucl. Phys. 18 (1974), 308.

[6] J. Bartels, H. Kowalski, A. Sabio-Vera, HERA-LHC Workshop, arXiv:hep-ph/0601012/13 (2006).

[7] CMS Collaboration, Expression of Interest submitted to the LHCC Meeting in January 2004 (2004).

[8] CMS Collaboration, TOTEM Collaboration, Prospects for Diffractive and Forward Physics at the LHC, CERN-LHCC-2006-039/G-124 (2006), CMS Note-2007/002, TOTEM Note 06-5.

[9] K.Borras for the SMIX Initiative, HERA-LHC Workshop 2007, http://indico.cern. ch/materialDisplay.py?contribId=137\&; sessionId=24\&; materialId=slides\&; conf $I d=11784$. 